\title{
Vertex Decomposable Simplicial Complexes Associated to Path Graphs
}

\author{
Somayeh Moradi ; \\ Department of Mathematics, School of Science, Ilam University, Ilam, Iran \\ Received: 3 March 2017 Accepted: 3 April 2018 \\ Extended Abstract \\ Paper pages (79-84)
}

Introduction

Vertex decomposability of a simplicial complex is a combinatorial topological concept which is related to the algebraic properties of the Stanley-Reisner ring of the simplicial complex. This notion was first defined by Provan and Billera in 1980 for k-decomposable pure complexes which is known as vertex decomposable when $k=0$. Later Bjorner and Wachs extended this concept to non-pure complexes. Being defined in an inductive way, vertex decomposable simplicial complexes are considered as a well behaved class of complexes and has been studied in many research papers. Because of their interesting algebraic and topological properties, giving a characterization for this class of complexes is of great importance and is one of the main problems in combinatorial commutative algebra. In this regard obtaining families of simplicial complexes with this property is of great interest. In this paper we present a new family of vertex decomposable simplicial complexes, which is associated to the t-clique ideal of the complement of path graphs. The t-clique ideal is a natural generalization of the concept of the edge ideal of a graph. For a graph $\mathrm{G}$, a complete subgraph of $\mathrm{G}$ with $\mathrm{t}$ vertices is called a $\mathrm{t}-$ clique of $\mathrm{G}$. The ideal $K_{t}(G)$ generated by the monomials $x_{i_{1}} \cdots x_{i_{t}}$ of degree $\mathrm{t}$ such that the induced subgraph of $\mathrm{G}$ on the set $\left\{x_{i_{1}}, \ldots, x_{i_{t}}\right\}$ is a complete graph, is called the t-clique ideal of G. We consider the Stanley- Reisner simplicial complex of the ideal $K_{t}\left(P_{n}^{c}\right)$, where $P_{n}$ is a path graph of order $\mathrm{n}$. For such a simplicial complex $\Delta$, we obtain the set of facets of $\Delta$ and using this characterization we show that every such simplicial complex is vertex decomposable, whose shedding vertex is an endpoint of the path graph. Indeed, any simplicial complex in this family is Cohen-Macaulay, since it is pure. Since edge ideals of graphs are in fact 2-clique ideals, this family of simplicial complexes contains the independence complexes of complement of path graphs. Finally, as a consequence it is shown that the t-independence ideal of the complement of a path graph is vertex splittable and its Betti splitting is presented

\section{Material and methods}

To prove the vertex decomposability of $\Delta_{K_{t}\left(P_{n}^{c}\right)}$, first we characterize the set of facets of $\Delta_{K_{t}\left(P_{n}^{c}\right)}$. This helps us to find a shedding vertex for this simplicial complex and then by an inductive approach the vertex decomposability has been proved.

\section{Results and discussion}

For positive integers $n$ and $t$, we show that a subset $\mathrm{F}$ of the vertex set of $P_{n}$ is a facet of $\Delta_{K_{t}\left(P_{n}^{c}\right)}$ if and only if $|F|=2 t-2$ and every component of the induced subgraph $P_{n}[F]$ is a 
path graph of even order. Using this characterization, it is shown that any endpoint of the path graph is a shedding vertex of $\Delta_{K_{t}\left(P_{n}^{c}\right)}$ and $\Delta_{K_{t}\left(P_{n}^{c}\right)}$ is vertex decomposable. Moreover, it is proved that the ideal $\left(K_{t}\left(P_{n}{ }^{c}\right)\right)^{\vee}$ has a Betti splitting.

\section{Conclusion}

The following conclusions were drawn from this research.

- A characterization for the set of facets of the simplicial complex $\Delta_{K_{t}\left(P_{n}^{c}\right)}$ is presented.

- The simplicial complex $\Delta_{K_{t}\left(P_{n}^{c}\right)}$ is vertex decomposable for any positive integers $n$ and $t$.

- The ideal $\left(K_{t}\left(P_{n}{ }^{c}\right)\right)^{\vee}$ has a Betti splitting for any any positive integers $n$ and $t$.

Keywords: T-clique ideal, Vertex decomposability, Path graph, Simplicial complex.

${ }^{*}$ Corresponding author: $\quad$ somayeh.moradi1@gmail.com 